\title{
Bewertung von Wohlbefinden in der praktischen Nutztierhaltung - Diskussion der Kriterienauswahl am Beispiel Mastschweinehaltung
}

\author{
RAGNHILD E. F. WEBER-JONKHEER und ANNE VALLE ZÁRATE
}

Institut für Tierproduktion in den Tropen und Subtropen, Universität Hohenheim, Stuttgart, Deutschland

\section{Zusammenfassung}

Für die Einschätzung des Wohlbefindens von Tieren unter praxisüblichen Haltungsbedingungen spielt - neben einer klaren Definition von Wohlbefinden - die Auswahl der Indikatoren eine entscheidende Rolle. Am Beispiel Mastschweinehaltung wird die Eignung von Kriterien aus der Physiologie, der Immunologie und Pathologie, der Ethologie sowie technischer Eigenschaften der Haltungsbedingungen diskutiert. Während physiologische und immunologische Parameter unter kontrollierten Bedingungen Erkenntnisse über Stressreaktionen liefern können, ist deren Übertragung auf Praxisbedingungen kaum möglich. Dagegen lassen sich ethologische Indikatoren als äußere Zeichen innerer Zustände in der Praxis am zuverlässigsten erfassen. Neben Stereotypien und Verhaltensstörungen als deutliche Zeichen für vermindertes Wohlbefinden, insbesondere in reizarmer Haltungsumwelt, kann die Ausführung speziesspezifischer Verhaltensweisen auf einen positiven Einfluss auf das Wohlbefinden hinweisen. Verhaltensbeobachtungen über längere Zeiträume unter Berücksichtigung einer Vielzahl an (ethologischen) Indikatoren bilden eine solide Basis in der Methodenauswahl. Für die weitere Interpretation der Ergebnisse sollten die ethologischen Daten durch Parameter ergänzt werden, die ohne größere Störungen der Tiere zu erfassen sind: Allgemeinbefinden der Tiere inklusive Verletzungen und Verschmutzung, Leistung und technische Größen der Haltungsbedingungen sowie die zunehmend an Bedeutung gewinnende Evaluierung aus Schlachtkörperuntersuchungen.

Schlüsselwörter: Wohlbefinden, Wohlergehen, Kriterien, Nutztierhaltung, Schwein

\section{Abstract}

\section{Evaluating welfare in practical farm animal husbandry - discussion of criteria selection using the example of fattening pig husbandry}

In the evaluation of animal welfare under practical husbandry conditions - beside a clear definition of welfare - selection of indicators plays an important role. Using the example of fattening pig husbandry, the usefulness of physiological, immunological and pathological, ethological, and technical criteria of husbandry conditions is discussed. While physiological and immunological parameters can lead to findings about stress reactions under controlled circumstances their direct transfer to practical conditions is hardly possible. On the contrary, ethological indicators as outer signs of inner states can 
be recorded most reliable. Beside stereotypies and aberrant behaviour as clear signs of reduced welfare, especially in barren environments, the performance of species-specific behaviour can point to a positive influence on welfare. Behavioural observations over a longer period of time considering plenty of (ethological) indicators build a solid base for selection of methods. For further interpretation of results behavioural data should be supplemented by parameters which can be recorded without major distraction of the animals: general condition of the animal including injuries and soiling, performance, technical data of the husbandry conditions as well as the increasingly important evaluation of carcasses.

Keywords: welfare, well-being, criteria, animal husbandry, pig

\section{Einleitung}

In Deutschland wird der überwiegende Teil an Mastschweinen aufgrund betriebswirtschaftlicher Vorteile in strohlosen Haltungssystemen gehalten (ZDS 1998, ZDS 2005). Reichhaltig ausgestaltete Haltungssysteme sind zwar arbeitswirtschaftlich und im Hinblick auf die tierischen Leistungen weiterentwickelt worden, können sich aber aufgrund betriebswirtschaftlicher Erwägungen nicht in der Praxis durchsetzen (AMON et al. 2001, AREY und SANCHA 1996, BAUMGARTNER et al. 1993, HÖRNING 1991, MAIER et al. 1992, PFLANZ 2007, WECHSLER 1992). Man kann davon ausgehen, dass große Einschränkungen des arteigenen Verhaltens, z.B. durch Reizarmut oder Enge, das Wohlbefinden einschneidend reduzieren, indem sie positives Verhalten (z.B. Spielen) vermindern und durch Verhaltensstörungen neben Verletzungen und Schäden auch Frustration, Angst oder Langeweile und damit Leiden bei den Tieren auslösen können (BAUMGARTNER et al. 2005, BEATTIE et al. 1995, BODENKAMP 1998, BÖHMER und HOY 1994, DE JONG et al. 1998, FRASER et al. 1991, JACKISCH et al. 1996, KRÖTZL et al. 1994, LAY JR. et al. 2000, LYONS et al. 1995, SIEGWART et al. 2005, STUBBE 2000, VAN ROOIJEN 1984, 1991). Somit besteht unter praxisüblichen Haltungsbedingungen ein erheblicher Zielkonflikt zwischen dem Wohlbefinden von Mastschweinen und der Erfordernis rentabler Produktion. Daraus erwachsen zahlreiche Bemühungen, praxisgerechte Haltungsbedingungen hinsichtlich ihrer Tiergerechtheit zu bewerten und tiergerechter zu gestalten (BERTRAM und HERRMANN 1998, BMVEL 2005, GAULY et al. 2006, PFLANZ 2007, WECHSLER 2005). In Deutschland spiegelt sich dies beispielsweise in der neu gefassten Tierschutz-Nutztierhaltungsverordnung wider, in der dem Erkundungsverhalten von Schweinen Rechnung getragen wird (BGBL 2006). Um einschätzen zu können, inwieweit Wohlbefinden der Tiere unter Praxisbedingungen gegeben sein kann, bedarf es neben einer klaren Definition (WEBER und VALLE ZÁRATE 2005) einer Auswahl an Kriterien. Der Beitrag diskutiert die Auswahl geeigneter Kriterien zur Schätzung von Wohlbefinden unter Praxisbedingungen am Beispiel der Mastschweinehaltung. Dies soll einen Beitrag dazu leisten, die Förderung von Wohlbefinden in der praktischen Nutztierhaltung auf eine methodisch nachvollziehbare Grundlage zu stellen. 


\section{Indikatoren für Wohlbefinden}

Indikatoren lassen sich in mehrere Bereiche einteilen, sie lassen sich der Physiologie, der Immunologie und Pathologie und der Ethologie zuordnen. Darüber hinaus werden technische Haltungsbedingungen in Bezug auf Tiergerechtheit beschrieben.

\section{Physiologische Indikatoren und Leistung}

Physiologische Indikatoren stammen hauptsächlich aus zwei körpereigenen Systemen, dem Hypothalamus-Hypophysen-Nebennierenrinden System (HPA) und dem SympathoNebennierenmark System (SA) (VON BORELL 2000). Während die Aktivierung des HPASystems eher längerfristige Anpassungen widerspiegelt, reagiert das SA-System schneller in Ausbildung des kurzfristigen Kampf- und Fluchtsyndroms (VON BORELL 2000). Indikatoren aus dem SA-System sind hauptsächlich Herzschlagfrequenz und -variabilität und Katecholaminkonzentrationen (Adrenalin- und Noradrenalin) (DE JONG et al. 1998, HANSEN und VON BORELL 2000, MARCHANT et al. 1995, MARCHANT et al. 1997, OTTEN et al. 1997, ROBERT et al. 1997). Im HPA-System gilt von den Glucocorticosteroiden der Nebennierenrinde vor allem Cortisol als Stressindikator (BARNETT et al. 1996, BEATTIE et al. 2000, BELLMANN et al. 2006, JANSSENS et al. 1995, KÜNZL und SACHSER 2000, OTTEN et al. 1997, OTTEN et al. 2000). Stress kann zu einer Minderung des Wohlbefindens führen, wobei die Grenze der akzeptablen Belastung für ein Tier umstritten ist (BARNETT und HEMSWORTH 1990). Diese Problematik spiegelt sich auch in den unterschiedlichen Definitionskonzepten von Stress wider. Nach VON BORELL (2000) beschreibt Stress »einen Zustand des Organismus', der durch spezifische Anpassungsreaktionen auf verschiedenartige Belastungsreize (Stressoren) gekennzeichnet ist«. TERLOUW et al. (1997) definieren Stress als den Zustand des Tieres, in dem seine ethologische und physiologische Anpassungsfähigkeit bereits überschritten ist.

Allen Untersuchungen, in denen physiologische Parameter als Stressindikatoren dienen, ist gemein, dass es sich um Versuchsanstellungen mit akuten Belastungssituationen oder (chronischen) Stress induzierenden Faktoren wie Anbindung oder rationierte Fütterung handelt.

Die Blutprobennahme selbst kann weiteren Stress verursachen (DUBREUIL et al. 1993), dazu liegen jedoch unterschiedliche Ergebnisse vor, was von der Blutentnahmetechnik und -dauer abhängig ist (ARKENAU 1996, DUBREUIL et al. 1990, LINK 1993). Untersuchungen zur Messung der Cortisolkonzentration im Speichel versuchen dieses Problem zu reduzieren (BROOM et al. 1995, EKKEL et al. 1995, PEETERS et al. 2006). Da nur unvollständige Übereinstimmung zwischen Plasma- und Speichelcortisolkonzentration besteht, kann die Interpretation der Ergebnisse erschwert sein (SCHÖNREITER und ZANELLA 2000, SIARD et al. 1997). Katheterisierung kann zur Stressminderung bei der Blutentnahme durchgeführt werden. Diese Methode ist sehr aufwendig (ARKENAU 1996). Darüber hinaus ist zu beachten, dass das Tragen der Katheter Stress bei der Blutentnahme nicht vollständig vermeidet und eine Beeinflussung sowohl physiologischer Werte (SCHOLZ 1981) als auch des Verhaltens der Tiere bewirken kann.

Eine Reihe weiterer physiologischer Indikatoren wie Konzentration und Variation von Prolactin, Wachstumshormon, Opioiden u.a. können zur Bestimmung von Stressreaktionen herangezogen werden (RUSHEN et al. 1990, RUSHEN et al. 1993, ZANELLA und BROOM 
1993). MANTEUFFEL und PUPPE (1997) urteilen, dass die Analyse von Hirnfunktionen den direktesten Zugang zum subjektiven Empfinden eines Tieres ermögliche. Dabei zeichnet sich nach neueren Erkenntnissen ab, dass Kerngebiete des limbischen Systems für die subjektive Bewertung von Sinneseindrücken eine entscheidende Rolle spielen. Dem limbisch-präfrontalen System mit seinen Neurotransmittern kommt für die Entstehung von Befindlichkeiten eine integrierende Bedeutung zu (TEUCHERT-NOODT 2004). Die Erfahrung bewusster Gefühle und die Fähigkeit der Kontrolle über emotionale Reaktionen sind in höchstem Maß von Interaktionen zwischen Hirnstammregionen und Neocortex als stammesgeschichtlich junger Teil der Großhirnrinde abhängig. Dabei werden bei Säugetieren Komplexität und Vielfalt höherer Funktionen wie Kognition, Bewusstsein, Emotion, Lernen u.a. vom Entwicklungsstand der Neocortex bestimmt (KENDRICK 2007).

Messungen von Hirnfunktionen wie auch anderer physiologischer Indikatoren müssen unter definierten, genau kontrollierbaren und daher stark eingeschränkten Bedingungen durchgeführt werden, um geeignete Referenzsituationen zu erstellen (MANTEUFFEL und PUPPE 1997). Eine Übertragung auf praktische Haltungsbedingungen mit ihren mannigfachen Einflüssen auf das Tier ist außerordentlich schwierig, wenn nicht unmöglich (MANTEUFFEL und PUPPE 1997). Physiologische oder auch Verhaltensindikatoren in standardisierten Tests sind oft nicht eindeutig einer Emotion zuzuordnen, da kognitive Prozesse an der individuellen Bewertung eines Reizes beteiligt sind (MENDL und PAUL 2005). Auch ist die Verbindung von Kognition und Emotion noch wenig erforscht. MENDL (1999) stellt fest, dass die größte Schwierigkeit in der Interpretation von Versuchsergebnissen zu kognitiven Funktionen in der Übertragung auf Praxisbedingungen liege. Die unterschiedlichen Einflüsse verschiedener Stressoren und ihr Zusammenwirken seien häufig Ursache für die widersprüchlichen Ergebnisse aus Untersuchungen physiologischer Indikatoren (MASON und MENDL 1993, MENDL 1999).

Die Wirkung von Langzeitstress, d.h. auch Stress, der unter Haltungsbedingungen entstehen kann, ist noch nicht hinreichend erforscht (RUSHEN 1991, TERLOUW et al. 1991). Chronischer Stress ist kein permanenter Zustand, sondern eher eine Zeitspanne mit periodisch wirkendem Stress, und kann nicht durch Veränderungen im HPA- oder SASystem gemessen werden, da aufgrund von Rückkopplungsmechanismen längerfristig eine »Normalisierung « eintritt. Allerdings kann die Regulierung dieser Systeme verändert sein, wodurch die Reaktion auf einen zusätzlichen akuten Stressor Sensibilisierung oder Desensibilisierung anzeigen kann (BEATTIE et al. 2000, BECKER et al. 1985, JENSEN et al. 1995b).

Zusammenfassend zeigt sich, dass physiologische Parameter weniger geeignet sind, die vielfältig bedingten Auswirkungen von Haltungsbedingungen in der Praxis abzubilden, ohne selbst einen zusätzlichen Stressfaktor darzustellen. Mit ihnen können vielmehr Reaktionen auf definiert eingegrenzte Stresssituationen untersucht werden (VON BORELL et al. 1995).

Die Auffassung, dass gute oder hohe Leistung für gutes Wohlbefinden ein hinreichender Indikator sei, wird nur noch selten vertreten. Hohe Leistung muss nicht unbedingt hohes Wohlbefinden implizieren, wohingegen Leistungseinbrüche mit Stress und vermindertem Wohlbefinden einhergehen (BRACKE et al. 2007). Stress kann sich indirekt anhand der Leistung widerspiegeln (EKKEL et al. 1995). Hohe Belegdichten und 
Schwanzbeißen können sich in verminderter Leistung als Stressreaktion niederschlagen (BELLMANN et al. 2006). Auch extreme Klimabedingungen können zu einer Minderung des Wohlbefindens von Schweinen führen. Einen Hinweis kann die Futteraufnahme und Futterverwertung geben (ANDRESEN und REDBO 1999, BEHNINGER et al. 1997, HAHN et al. 1987, RINALDO und LE DIVIDICH 1991, VERSTEGEN et al. 1978). Somit kann gute Leistung zwar als ein notwendiges, nicht jedoch als ein hinreichendes Kriterium betrachtet werden.

Bei den Schlachtleistungsmerkmalen können vor allem Abweichungen in der Entwicklung von pH-Werten, Leitfähigkeit, Wasserhaltevermögen u.a. sowie das Auftreten von Fleischqualitätsmängeln PSE (blass, weich, wässrig) und DFD (dunkel, fest, trocken) im Nachhinein einen Hinweis auf erhöhten Stress der Schweine während Transport und Schlachtung geben (BÄRLOCHER 2006, PEETERS et al. 2006).

\section{Immunologische und pathologische Indikatoren}

Gesundheit und Krankheit sind ähnlich schwierig zu definieren wie Wohlbefinden. Erstens stellt sich die Frage, welche Faktoren zu vollständiger Gesundheit beitragen (RIST 1978) und zweitens lässt sich nicht eindeutig eine Grenze zwischen krank und gesund ziehen, weil beide Zustände fließend über subklinische zu klinischer Erkrankung ineinander übergehen. BROOM (2006) betrachtet Gesundheit als einen wichtigen Teil von Wohlbefinden und Krankheit einerseits als Ursache, andererseits als Folge geringen Wohlbefindens.

Für Gesundheit bzw. Krankheit als Teilaspekt von Wohlbefinden unter Praxisbedingungen wird vorwiegend der physische Bereich betrachtet, da mentale und psychische Gesundheit nur indirekt erfasst werden können. Es ist davon auszugehen, dass Schweine, die ein gestörtes Allgemeinbefinden aufzeigen, bereits in ihrem Wohlbefinden beeinträchtigt sind. Zur Erfassung des Allgemeinbefindens werden folgende Merkmale herangezogen: Anteilnahme oder Augenausdruck, Ernährungszustand, Fressverhalten, Ausgeglichenheit in der Entwicklung einer Gruppe von Tieren, Kotbeschaffenheit, Bewegung, Körperhaltung, Absonderung eines Tieres von der Gruppe, Lautäußerungen, Glanz des Haarkleids, Hautfarbe, Atmung sowie Auftreten von Husten oder Niesen (MORTON und GRIFFITHS 1985).

Klinisch diagnostizierbare Krankheiten implizieren allfällig eine Einschränkung des Wohlbefindens. BLAHA (2006) schlägt als gut zu vergleichendes Häufigkeitsmaß infektiöser Erkrankungen den Tierbehandlungsindex vor. Er basiert auf der Annahme, dass Tiere, die häufiger oder länger antibiotisch behandelt wurden, weniger gesund waren und berechnet die durchschnittliche Anzahl an Behandlungstagen pro Tier oder Mastgruppe. Nach der Schlachtung können die erweiterte Schlachtkörperadspektion, Geschlinge- und Organuntersuchung und die Zuordnung der Ergebnisse zu Einzeltieren und Betrieben auch subklinische Zustände aufdecken und Möglichkeiten für die Einschätzung der Tiergesundheit unter verschiedenen Managementbedingungen eröffnen (BELLMANN et al. 2006, BLAHA 1993, LINDQUIST 1974, NIELSEN und INGVARTSEN 2000, SCHIMMEL 1992). Informationen über Befunddaten gewinnen im Rahmen der risikoorientierten Schlachttier- und Fleischuntersuchung zunehmend an Bedeutung (ABL 2004). Eine Standardisierung der Organbefundung hat sich in der Praxis jedoch bisher nur in Ansätzen gezeigt (QS 2009). 
EKESBO (1981, 1984) war einer der Ersten, der darauf hingewiesen hat, dass auch beobachtete Verletzungen (BÄCKSTRÖM 1973) Indikatoren für vermindertes Wohlbefinden sein können. Darauf aufbauend wurde eine Reihe von Erfassungsbögen entwickelt und zur Beurteilung von Haltungssystemen in der Schweinehaltung eingesetzt (DE KONING 1985, GLOOR 1984, 1988, KRAUSE 1995, LYONS et al. 1995, MAYER 1999, SCHÄFERMÜLLER 1996, SOMMER et al. 1999, STUBBE 2000). So konnte beispielsweise das Auftreten von Verletzungen im Verlauf der Mast innerhalb Haltungssystem beschrieben und Verletzungshäufigkeiten zwischen Haltungssystemen verglichen werden (MAYER 1999). Bei Sauen in Anbinde- oder Kastenstandhaltung konnten durch das Haltungssystem verursachte Verletzungspunkte und Verletzungsfolgen stereotyper Bewegungen identifiziert werden (DE KONING 1985, GLOOR 1984, 1988, SOMMER et al. 1999). Klauenverletzungen und Gliedmaßenveränderungen können Rückschlüsse auf die Verletzungsgefahr durch den Stallboden zulassen (BEYER und WECHSLER 1999, GREIF 1982, HESSE et al. 1993, KELLY et al. 2000, SAVARY et al. 2005, SCHUSTER 1984, SEUFERT et al. 1980, SIEGWART et al. 2005, WITTE 1999). Die in Verbindung mit der Adspektion des Integuments erhebbare Verschmutzung der Tiere und die zusätzlich erfasste Buchtenverschmutzung können einen Hinweis auf gestörte thermoregulatorische und territorial-ethologische (Trennung von Kot- und Liegebereich) Möglichkeiten geben (BADERTSCHER und SCHNIDER 2002, BEATTIE et al. 1998, LEGGE 1992, SCHÄFER-MÜLLER 1996, SIEGWART et al. 2005, SIMONSEN 1990), die beide eine Minderung des Wohlbefindens darstellen. Darüber hinaus kann es zu hygienischen Problemen (Endound Ektoparasitenbefall) kommen (HOY et al. 1997). Leber- bzw. Kotuntersuchungen geben Aufschluss über Endoparasitenbefall (BADERTSCHER und SCHNIDER 2002). Eine umfangreiche Darstellung von Indikatoren aus dem Bereich Tiergesundheit sowie Tierverhalten ist bei VELARDE und GEERS (2007) spezifisch fürs Schwein zusammengestellt. Hier werden für jeden Indikator Grundlagen und Methodik beschrieben sowie Gültigkeit, Zuverlässigkeit und Anwendbarkeit bezogen auf den Einsatz unter Praxisbedingungen bewertet.

Die Krankheitsanfälligkeit kann durch ungünstige Umwelt- oder Haltungsbedingungen, z.B. Hitze oder Kälte, Enge oder Zusammenbringen fremder Tiere, steigen. Immunsuppression kann u.a. durch Testen der Immunantwort nach Antigenverabreichung, Bestimmen der Immunglobulinkonzentration, Leukozytenzahl oder Lymphozytenfunktion gemessen werden (BELLMANN et al. 2006, BROOM et al. 1995, BROOM 1996, DUBREUIL et al. 1993, KELLY et al. 2000, OTTEN et al. 2000, SIEGEL 1987, TURNER et al. 2000, ZANELLA et al. 1991). Die Immunsuppressionsreaktionen auf Belastungen (Stress) sind nicht konsistent, sondern abhängig von Genetik, Ernährungszustand, Antigenkonzentration u.a. (Siegel, 1987). Eng verbunden mit dem Immunsystem ist das (Zentral)nerven- und Hormonsystem, wobei Informationen wechselseitig ausgetauscht werden (ADER et al. 1995, TUCHSCHERER und MANTEUFFEL 2000, SIEGEL 1987, VON BORELL 2000). Durch die hohe Komplexität der Systeme ist es unmöglich, immuno-neuroendokrine Interaktionen vollständig zu analysieren (TUCHSCHERER und MANTEUFFEL 2000). In ihrem Übersichtartikel stellen TUCHSCHERER und MANTEUFFEL (2000) fest, dass bei landwirtschaftlichen Nutztieren Stress-Belastungsexperimente häufig zu widersprüchlichen Ergebnissen geführt haben oder kein Zusammenhang zwischen Stressfaktor und Immunparameter festgestellt 
werden konnte. Sie schlussfolgern, dass generell ein negativer Einfluss von Disstress auf die Immunfunktion als gesichert angesehen werden kann, spezifische Immunfunktionsreaktionen auf unterschiedliche Stressoren jedoch nicht vorausgesagt werden könnten. Dies liege vor allem an der subjektiven, von der individuellen Lerngeschichte beeinflussten, instinktiven und kognitiven Bewertung einer Stresssituation (TUCHSCHERER und MANTEUFFEL 2000) und an dem fließenden Übergang zwischen Eu- und Disstress. Auch bei neueren Untersuchungen bestätigen BELLMANN et al. (2006), dass Immunfunktionen wenig durch die Gestaltung der Haltungsumwelt beeinflusst werden. Andererseits geben Untersuchungen zur kognitiven Bereicherung eines Haltungssystems (Ton-Schalter-Futterautomat) Hinweise auf eine Verbesserung der Gesundheit (Immunglobulin G, Wundheilung) durch Eustress (PUPPE et al. 2005). Nur unter kontrollierten Bedingungen durchgeführte Untersuchungen können derzeit mehr Einblick in die Interaktionen zwischen verschiedenen Stressoren und dem Immunsystem bieten.

Mortalität kann insofern als Indikator dienen, als sie deutlich Missstände anzeigt und ihre Ursachen im Nachhinein untersucht und die Ergebnisse zur Vermeidung weiteren Sterbens anderer Individuen herangezogen werden können (FRERKING et al. 1996, LINDQUIST 1974, LOSINGER et al. 1998). Langlebigkeit ist ein weiterer Indikator für Wohlbefinden, der jedoch bei Mastschweinen keine Bedeutung hat.

\section{Ethologische Indikatoren}

Ethologische Indikatoren können einerseits auf Verhaltensstörungen oder Stereotypien begrenzt werden, andererseits können sie aus einem vollständigen Ethogramm stammen. Es wird unterschieden zwischen Indikatoren, die vermindertes Wohlbefinden anzeigen und solchen, die positive Anzeichen von Wohlbefinden sind. Ethologische Indikatoren für Wohlbefinden können sehr gut unter Praxisbedingungen eingesetzt werden, da sie unmittelbar erfassbar sind. Sie sind gewissermaßen äußere Zeichen innerer Zustände und Emotionen (SIGNORET und VIEUILLE 1996, VELARDE und GEERS 2007). Wertvolle und wichtige Aspekte bzgl. Versuchsplanung und statistischer Auswertung ethologischer Untersuchungen beschreiben WECHSLER (2004) und GYGAX (2004). Unter anderem wird dabei betont, dass die Auswahl der zu beobachtenden Verhaltensweisen der Fragestellung angemessen sein muss. Dies bedeutet auch, dass eine grobe Einteilung des Verhaltens z.B. nur in Stehen und Liegen nicht zu klaren Schlussfolgerungen führen kann (WECHSLER 2004).

Stereotype Verhaltensweisen scheinen ausschließlich bei Tieren in Tierhaltung aufzutreten (MASON 1991). Sie werden für Schweine am häufigsten bei Sauen unter Anbindung oder in Kastenständen, d.h. unter extremer Bewegungseinschränkung sowie in Verbindung mit dem Kontrollverlust der Schweine über das Fressen beschrieben. Dabei spielt vor allem das Vorlegen geringer Volumina gering strukturierten Futters eine entscheidende Rolle, wobei auch der Energiegehalt des Futters einen Einfluss haben kann (ARELLANO et al. 1992, BERGERON et al. 2000, BROOM et al. 1995, JENSEN et al. 1995a, ROBERT et al. 1997, SPOOLDER et al. 1995, VAN DIJK et al. 2000, VIEUILLE-THOMAS et al. 1995). Ob Stereotypien Indikatoren für verringertes Wohlbefinden sind oder lediglich einen Anpassungsmechanismus darstellen, ist umstritten (COOPER und NICOL 1993, MASON, 1991, RUSHEN 1993). Ursache und vor allem Wirkung von Stereotypien 
sind nicht eindeutig geklärt. Die physiologischen Korrelate zu stereotypem Verhalten sind komplex und abhängig von der Art der Stereotypie, der Art und Dauer des Stressors und der subjektiven Wahrnehmung der Umwelt durch das Tier (TERLOUW et al. 1991, WIEPKEMA et al. 1993). Somit ist die simple Hypothese, Stereotypien hätten einen narkotisierenden Effekt und erleichterten die Anpassung des Tieres an die (unzureichenden) Haltungsbedingungen nicht mehr haltbar (MASON 1991, RUSHEN et al. 1990). Sogar schon relativ primitive Tiere interagieren spontan und aktiv mit ihrer Umwelt und reagieren nicht nur auf Stimuli (WEMELSFELDER 1997). Dies schließt Subjektivität des Tieres ein und bedeutet, dass Tiere Erwartungen haben, die unter restriktiven Bedingungen zu Frustration, Langeweile oder Depression und Angst führen können. Auf der anderen Seite gibt es Beispiele dafür, dass durch die Unterstützung ihrer kognitiven Fähigkeiten das Wohlbefinden von Tieren in Tierhaltung verbessert werden kann. In einer Untersuchung von Absetzferkeln stellten DUDINK et al. (2005) fest, dass bereits die Erwartung bzw. Ankündigung des Angebots von Anreicherungen zu mehr Spielverhalten führte. PUPPE et al. (2007) beobachteten weniger gegenseitige Bauchmassage bei Mastschweinen, die sich ihr Futter aus einer Futterstation mit Rufton und Schalter erarbeiten mussten als bei Kontrolltieren, deren Haltungsumwelt keine Anreicherung auf Basis anhaltender kognitiver Herausforderung besaß, und werten dies als positiv für das Wohlbefinden. Durch die wiederholte, belohnte Bewältigung anspruchsvoller Verhaltensaufgaben hätten die Schweine eine positive Rückkopplung, die sowohl ihren Motivationsbedürfnissen entspräche (mehr Bewegung, mehr Kontrolle über die Haltungsumwelt, Belohnung und Verstärkung der Motivation) als auch durch die häufige Vorlage kleiner Futterportionen am Tag ihrem natürlichen Verhalten näher käme (PUPPE et al. 2007).

Das Auftreten von Stereotypien ist ein sicherer Indikator dafür, dass Haltungsbedingungen das Ausführen speziesspezifischen Verhaltens in speziesspezifischen Zeitanteilen nicht ausreichend ermöglichen und damit das Wohlbefinden stark eingeschränkt wird (MEYER 2004, WEMELSFELDER 1997).

Zu der Frage, inwieweit das gegenseitige Bearbeiten bei Schweinen einen positiven Aspekt von Sozialverhalten darstellt oder als eine Ersatzhandlung angesehen werden muss, besteht Uneinigkeit. MEYNHARDT (1990) beschreibt bei Wildschweinen ein gegenseitiges Absuchen der Tiere zur Entfernung von Fremdkörpern, während BRIEDERMANN (1990) keine Sozialpflege beim Wildschwein beobachtete. Bei den Verhaltensweisen Beknabbern, Belecken und Massieren von Artgenossen finden sich teilweise widersprüchliche Aussagen in der Literatur, sie werden jedoch überwiegend als Ersatzhandlungen oder Konfliktverhalten interpretiert (HANSEN und VON BORELL 2000, STUBBE 2000). Die in reizarmen Umgebungen häufiger beobachteten Bearbeitungen von Buchtengenossen oder Wurfgeschwistern deuten auf ein kompensatorisches Verhalten hin (BEATTIE et al. 1995, BODENKAMP 1998, BÖHMER und HOY 1994, BOLHUIS et al. 2005, DE JONG et al. 1998, FRASER et al. 1991, JACKISCH et al. 1996, JAKOB 1987, KRÖTZL et al. 1994, LAY JR. et al. 2000, LYONS et al. 1995, PEETERS et al. 2006, VAN DE WEERD et al. 2006, WEBER 2003). In reizarmer Umgebung wurde teilweise auch aggressives Verhalten (Kämpfen) häufiger beobachtet (BEATTIE et al. 1995, BODENKAMP 1998, BÖHMER und HOY 1994, JAKOB 1987, LAY JR. et al. 2000). Dabei ist der Einfluss der Raumgestaltung auf 
das partnerorientierte Verhalten der Schweine größer als der des Platzangebots (BEATTIE et al. 1996). Für die Bewertung der Verhaltensweisen Aggression und gegenseitige Manipulation gilt wie für die Stereotypien, dass eine Verringerung des Wohlbefindens der Tiere angenommen werden kann, zumal Verletzungen und Schmerzen bei den bearbeiteten oder angegriffenen Tieren entstehen können. MARCHANT et al. (1995) stellten bei Sauen eine erhöhte Herzfrequenz in kämpferischen Auseinandersetzungen, insbesondere bei den unterlegenen Tieren fest. HAGELSØ GIERSING und STUDNITZ (1996) sehen als Motivation von Hyperaggressivität Frustration, Angst oder negative Erfahrungen und schlechte Haltungsbedingungen, sodass sie sie als negativ für das Wohlbefinden auch beim angreifenden Tier beurteilen.

Passivität zählt ebenfalls als Indikator für verringertes Wohlbefinden. VAN ROOIJEN $(1984,1991)$ weist darauf hin, dass zu viel Vorhersagbarkeit von Haltungssystemen im Vergleich zu natürlicher Umgebung ein Problem von Stress durch Langeweile verursachen kann. In reizarmer Umgebung wurde bei Mastschweinen Inaktivität häufiger beobachtet (BEATTIE et al. 1995, BODENKAMP 1998, LAY JR. et al. 2000, LYONS et al. 1995, STUBBE 2000), ebenso bei Sauen in Abferkelbuchten mit Ferkelschutzkorb (AREY und SANCHA 1996). Die Gründe für Inaktivität können neben Reizarmut und Langeweile aber auch andere ungünstige Bedingungen wie glatte oder harte Böden, Verletzungen oder Beinschwäche oder Temperaturen oberhalb der oberen kritischen Temperatur sein (BEHNINGER et al. 1997, SAMBRAUS 1985, VAN ROIJEN 1991).

Neben den Verhaltensweisen, die als Indikatoren für verringertes Wohlbefinden dienen, lassen sich auch Verhaltensweisen auf Grundlage eines vollständigen Ethogramms als positive Indikatoren für Wohlbefinden nutzen (HÖRNING 1991). Ein Ethogramm kann unter wenig restriktiven Bedingungen erstellt werden. Für Hausschweine gibt es bereits ethologische Untersuchungen unter semi-natürlichen Bedingungen, die umfangreiches Wissen über das Verhaltensrepertoire der domestizierten Tiere geben (JENSEN 1986, JENSEN und STANGEL 1992, STOLBA 1984, STOLBA und WOOD-GUSH 1984, 1989). Unter restriktiven Haltungsbedingungen in der Praxis können einige Verhaltensweisen gar nicht ausgeführt werden, viele werden stark behindert (HÖRNING 1998), Verhaltensstörungen können zusätzlich auftreten. Umgekehrt gibt das Auftreten beispielsweise speziesspezifischen Körperpflege-, Erkundungs- und Ruheverhaltens in den jeweiligen spezifischen Zeitanteilen einen Hinweis darauf, dass diese Verhaltensweisen in einer Haltungsumwelt möglich sind und das Wohlbefinden der Tiere positiv beeinflussen. Dabei werden nicht alle Verhaltensweisen als gleichermaßen relevant bewertet (z.B. Sexualverhalten bei Mastschweinen).

Spielverhalten ist ein deutlicher Indikator für Wohlbefinden (DUDINK et al. 2005). Spielen ist gekennzeichnet durch das Fehlen des Ernstbezugs, d.h. Verhaltensabläufe erfolgen teilweise spontan ohne Reiz-Reaktions-Beziehung in nicht funktionalem Zusammenhang und in freier Kombination und zeitlicher Abfolge (GRAUVOGL 2000). Obwohl insbesondere bei Jungtieren ein Spieltrieb besteht (GRAUVOGL 2000), wird Spiel erst ausgeführt, wenn Tiere kein anderes überlebensnotwendiges Verhalten ausführen müssen (GRAUVOGL 2000). Daher kann es als Zeichen von Wohlbefinden und Bedürfnisbefriedigung dienen. Beispiele hierfür sind umgekehrt darin zu sehen, dass kranke, verletzte oder apathische, hungrige oder bedrohte Tiere nicht spielen (GRAUVOGL 2000). 
Darüber hinaus ist Spielverhalten bei ungünstigen Haltungsbedingungen wie Platzmangel, ungünstigen Umgebungstemperaturen, glatten Böden und reizarmer Aufstallung reduziert (BOLHUIS et al. 2005, JENSEN und KYHN 2000, JONES und NICOL 1998).

\section{Technische Indikatoren für Tiergerechtheit}

Eine Beschreibung von Haltungssystemen anhand technischer Indikatoren kann dazu dienen, Verletzungen, Krankheiten und Verhaltensstörungen vorzubeugen. Andererseits kann das Management von Haltungssystemen einen sehr großen Einfluss auf die Tiergerechtheit haben (PFLANZ 2007). BRACKE et al. (2004a, 2004b) kombinierten beschreibende Haltungssystem- und Managementattribute, um daraus ein computergestütztes System zu entwickeln, das unter Berücksichtigung von Wichtungsfaktoren einen Risikofaktor für Schwanzbeißen bei Mastschweinen kalkuliert. Das Programm soll dazu dienen, das Risiko für Schwanzbeißen unter Praxisbedingungen vorherzusagen sowie Schwanzbeißen vorzubeugen und möglicherweise zu verhindern. Der so genannte Nationale Bewertungsrahmen Tierhaltungsverfahren (KTBL 2006) beschreibt 139 Haltungsverfahren für verschiedene Tierarten und ordnet diesen - neben Umweltwirkungen wissenschaftliche Erkenntnisse und gesicherte praktische Erfahrungen zur Einschätzung der Risiken für die Tiergesundheit und die Auswirkungen auf das Tierverhalten zu. In den Fällen, in denen keine entsprechenden ausreichenden Informationen vorlagen, wurden grundlegende Erkenntnisse auf einzelne Haltungsverfahren übertragen oder Experteneinschätzungen zugrunde gelegt. Für den Bereich Tierverhalten wird eingeschätzt, inwieweit die jeweils baulich-technischen Ausgestaltungen der Haltungsverfahren das Verhalten auf Grundlage eines vollständigen Ethogramms einschränken. Diese Einschätzung erfolgt in drei Abstufungen je einbezogene Verhaltensweise (aus unterschiedlichen Funktionskreisen) und wird ohne Wichtung summiert und in einer Gesamtbewertung in wiederum drei Abstufungen zusammengefasst. Stereotypien und Verhaltensstörungen werden im Bereich Tiergesundheit aufgeführt. Die Teilbewertungen aus den Bereichen Tiergerechtheit (bestehend aus Tierverhalten und Tiergesundheit) und Umweltwirkungen werden zu einem Gesamtergebnis integrativ zusammengezogen, das sich allerdings nicht rechnerisch aus den Teilergebnissen ergibt, sondern eine gemeinsame Meinung der Experten aus den Teilbereichen wiedergibt. Die Wichtung und Beurteilung von Einzelfaktoren integrierender Systeme ist umstritten (VELARDE und GEERS 2007). Während in der Praxis schnell einsetzbare Beurteilungssysteme zum Vergleich von Haltungssystemen genutzt werden, die nur eine punktuelle Situation erfassen können, sollten für die Schätzung des Wohlbefindens der Tiere in einem Haltungssystem längerfristige Auswirkungen auf die Tiere Berücksichtigung finden.

\section{Schlussfolgerungen zur Auswahl der Indikatoren für die Schätzung von Wohlbefinden unter Praxisbedingungen}

Für die Schätzung von Wohlbefinden unter Praxisbedingungen, die mit einer Vielzahl sich gegenseitig beeinflussender Faktoren langfristig auf die Tiere wirken, eignen sich besonders ethologische Indikatoren als äußere Zeichen innerer Zustände. Verhaltensbeobachtungen über längere Zeiträume (z.B. Mastperiode) sind dafür geeignet, das 
»ungestörte« Verhalten der Schweine unter Praxisbedingungen zu beurteilen. Die Einbeziehung von Verhaltensweisen, die im Hinblick auf Wohlbefinden negativ zu beurteilen sind, neben solchen, die positiv Wohlbefinden der Tiere anzeigen, führt dazu, dass die Haltungsbedingungen nicht nur dahin gehend beurteilt werden können, inwieweit sie negative Auswirkungen auf das Wohlbefinden der Tiere verursachen, sondern auch für das Wohlbefinden positives Verhalten zulassen. Im Gegensatz dazu sind, abgesehen von der Erfassung der Leistung der Tiere, physiologische Indikatoren wenig geeignet, da sie unter eingegrenzten, standardisierten Versuchsbedingungen erfasst werden müssen und einen zusätzlichen Stressor darstellen können. Analoges gilt für die Parameter des Immunsystems. Dagegen können Allgemeinbefinden, Verletzungen und Verschmutzungen mittels Tierkontrolle ohne größere Beeinflussungen der Tiere über den üblichen Management bedingten Umgang hinaus erfasst werden. Subklinische Zustände können nach der Schlachtung weiteren Aufschluss über die Tiergesundheit geben. Verletzungen des Integuments, der Gliedmaßen und der Klauen der Tiere können Rückschlüsse auf mögliche haltungsbedingte Ursachen zulassen. Technische Indikatoren dienen unterstützend der Ursachenforschung beim Auftreten von Verletzungen, Erkrankungen oder Verhaltensstörungen. Die Auswahl und Berücksichtigung von nur sehr wenigen Indikatoren führt zu einer verzerrten Beurteilung. Daher sollten so viele Indikatoren wie möglich und von der Zielsetzung der Untersuchung und statistischen Auswertung sinnvoll einbezogen werden.

\section{Literatur}

ABL (Amtsblatt der Europäischen Union) Verordnung (EG) Nr 853/2004 des Europäischen Parlaments und des Rates vom 29. April 2004 mit spezifischen Hygienevorschriften für Lebensmittel tierischen Ursprungs. ABI. L 139, 30.04.2004, 55-205

Ader R, Cohen N, Felten D (1995) Psychoneuroimmunology interactions between the nervous system and the immune system. Lancet 345, 99-103

Amon T, Jeremic D, Gallob M, Amon B, Harrich K, Bauer E, Boxberger J (2001) Schweinefleischerzeugung im Stolba-Familienstall. Proc 6. Wissenschaftstagung zum Ökologischen Landbau. Von Leit-Bildern zu Leit-Linien, Freising-Weihenstephan, 6.-8.3.2001, 313-6

Andresen N, Redbo I (1999) Foraging behaviour of growing pigs on grassland in relation to stocking rate and feed crude protein. Appl Anim Beh Sci, 183-97

Arellano PE, Pijoan C, Jacobson LD, Algers B (1992) Stereotyped behaviour, social interactions and suckling pattern of pigs housed in groups or in single crates. Appl Anim Beh Sci 35, 157-66

Arey DS, Sancha ES (1996) Behaviour and productivity of sows and piglets in a family system and in farrowing crates. Appl Anim Beh Sci 50, 135-45

Arkenau EF (1996) Systematische Einflussfaktoren auf Blutparameter von Sauen unter Stallhaltungsbedingungen mit Stroheinsatz. Diss Uni Kiel, SR Inst Tierzucht und Tierhaltung, Uni Kiel 93

Bäckström L (1973) Environment and animal health in piglet production. A field study of incidences and correlations. Acta Vet Scand 14 Suppl 41, 1-240

Badertscher R, Schnider R (2002) Vollspaltenbodenställe und Systeme mit Einstreu und Auslauf für Mastschweine. FAT-Berichte 585, Tänikon

Bärlocher HU (2006) Influence of alternative semi-outdoor housing systems in comparison with the conventional indoor housing on carcass composition and meat and fat quality of finishing pigs. SR FAT 71, Tänikon

Barnett JL, Hemsworth PH (1990) The validity of physiological and behavioural measures of animal welfare Appl Anim Beh Sci 25, 177-87 
Barnett JL, Cronin GM, McCallum TH, Newman EA, Hennessy DP (1996) Effects of grouping unfamiliar adult pigs after dark, after treatment with amperozide and by using pens with stalls, on aggression, skin lesions and plasma cortisol concentrations. Appl Anim Beh Sci 50, 121-33

Baumgartner J, Heizmann, V Krejci C (1993) Zum Einfluss des Absetzens auf das Verhalten juveniler Hausschweine in einem modifizierten Stolba-Familienstall. Akt Arb z artgem Tierhltg 1992, KTBL $356,141-54$

Baumgartner J, Verhovsek D, Troxler J (2005) Verhalten, haltungsbedingte Schäden und biologische Leistungen von Sauen in drei Typen von Abferkelbuchten. Akt Arb z artgem Tierhltg 2005, KTBL 441, 265-73

Beattie VE, Walker N, Sneddon IA (1995) Effects of environmental enrichment on behaviour and productivity of growing pigs. Anim Welf 4, 207-20

Beattie VE, Walker N, Sneddon IA (1996) An investigation of the effect of environmental enrichment and space allowance on the behaviour and production of growing pigs. Appl Anim Beh Sci 48, 151-8

Beattie VE, Walker N, Sneddon IA (1998) Preference testing of substrates by growing pigs. Anim Welf 7 , 27-34

Beattie VE, O'Connell NE, Kilpatrick DJ, Moss BW (2000) Influence of environmental enrichment on welfare-related behavioural and physiological parameters in growing pigs. Anim Sci 70, 443-50

Becker BA, Nienaber JA, Christenson RK, Manak RC, DeShazer JA, Hahn GL (1985) Peripheral concentrations of cortisol as an indicator of stress in the pig. Am J Vet Res 46, 1034-8

Behninger S, Haidn B, Schön H (1997) Außenklimaställe für Mastschweine - Untersuchungsergebnisse zu Stallklima, Tierverhalten und Leistungsparametern. In: Proc 3. Int Tagung Bau, Technik und Umwelt in der landw. Nutztierhaltung, Kiel 11.-12.3.1997, 121-9

Bellmann O, Kanitz E, Tuchscherer M, Reinhart E, Ender K (2006) Wirkung eines homöopathischen Kombinationsarzneimittels auf Tiergesundheit und Leistung bei Mastschweinen. Arch Tierz 49, 370-81

Bergeron R, Bolduc J, Ramonet Y, Meunier-Salaün MC, Robert S (2000) Feeding motivation and stereotypies in pregnant sows fed increasing levels of fibre and/or food. Appl Anim Beh Sci 70, 27-40

Bertram HH, Herrmann HJ (1998) Konzepte der freiwilligen DLG-Prüfung in Deutschland. Beurteilung der Tiergerechtheit von Haltungssystemen, KTBL 377, 87-91

Beyer S, Wechsler B (1999) Einfluss der Spaltenbreite und des Bodentypes bei teilperforierten Böden auf die Klauengesundheit von Absetzferkeln. In: Proc 14. IGN 6. Freiland Tagung Tierhaltung und Tiergesundheit, Wien 29.9.-1.10.1999, 120-3

BGBI (Bundesgesetzblatt) (2006) Verordnung zum Schutz landwirtschaftlicher Nutztiere und anderer zur Erzeugung tierischer Produkte gehaltener Tiere bei ihrer Haltung (Tierschutz-Nutztierhaltungs verordnung - TierSchNutztV). BGBI I 41, 31.08.2006

Blaha T (1993) Erfassung pathologisch-anatomischer Organbefunde am Schlachthof. Fleischwirtschaft $73,877-81$

Blaha T (2006) Auswirkungen des Paradigmenwechsels im Europäischen Lebensmittelrecht auf die Arbeit des praktizierenden Tierarztes. Der prakt Tierarzt 87, 639-43

BMVEL (Bundesministerium für Verbraucherschutz, Ernährung und Landwirtschaft) (2005) Zukunft der Nutztierhaltung Gutachten des Wissenschaftlichen Beirates Agrarpolitik, nachhaltige Landbewirtschaftung und Entwicklung ländlicher Räume beim BMVEL Berlin

Bodenkamp K (1998) Vergleichende Untersuchungen zur Haltung von Mastschweinen auf Kompost- und Vollspaltenboden. Diss Uni Kiel, SR Inst Tierzucht und Tierhaltung, Uni Kiel 107

Böhmer M, Hoy S (1994) Untersuchungen zum agonistischen Verhalten, zur Beschäftigung und zum Abliegeverhalten von Mastschweinen bei Haltung auf Tiefstreu mit mikrobiell enzymatischer Einstreubehandlung bzw auf Vollspaltenboden. Akt Arb z artgem Tierhltg 1993, KTBL 361, 264-73

Bolhuis JE, Schouten WGP, Schrama JW, Wiegant VM (2005) Behavioural development of pigs with different coping characteristics in barren and substrate-enriched housing conditions. Appl Anim Beh Sci 93, 213-28

Bracke MBM, Hulsegge B, Keeling L, Blokhuis HJ (2004a) Decision support system with semantic model to assess the risk of tail biting in pigs. 1. »Modelling «. Appl Anim Beh Sci 87, 31-44

Bracke MBM, Hulsegge B, Keeling L, Blokhuis HJ (2004b) Decision support system with semantic model to assess the risk of tail biting in pigs. 2. „Validation«. Appl Anim Beh Sci 87, 45-54

Bracke MBM, Zonderland JJ, Bleumer EJB (2007) Expert consultation on weighting factors of criteria for assessing environmental enrichment materials for pigs. Appl Anim Beh Sci 104, 14-23

Briedermann, L (1990) Schwarzwild. Dt. Landwirtschaftsverlag, Berlin

Broom DM (1996) A review of animal welfare measurement in pigs. Pig News and Info 17, 109N-14N

Broom DM (2006) Behaviour and welfare in relation to pathology. Appl Anim Beh Sci 97, 73-83 
Broom DM, Mendl MT, Zanella AJ (1995) A comparison of the welfare of sows in different housing conditions. Anim Sci 61, 369-85

Cooper, JJ, Nicol, CJ (1993) The »coping" hypothesis of stereotypic behaviour a reply to Rushen. Anim Beh 45, 616-8

De Jong IC, Ekkel ED, van de Burgwal JA, Lambooij E, Korte SM, Ruis MAW, Koolhaas JM, Blokhuis HJ (1998) Effects of strawbedding on physiological responses to stressors and behavior in growing pigs. Physiol Beh 64, 303-10

De Koning R (1985) On the well-being of dry sows. Diss Uni Utrecht

Dubreuil P, Couture Y, Tremblay A, Martineau G-P (1990) Effects of experimenters and different blood sampling procedures on blood metabolite values in growing pigs. Can J Vet Res 54, 379-82

Dubreuil P, Farmer C, Couture Y, Petitclerc D (1993) Hematological and biochemical changes following an acute stress in control and somatostasin-immunized pigs. Can J Anim Sci 73, 241-52

Dudink S, de Jonge FH, Spruijt BM (2005) Announcing the arrival of enrichment increases play behaviour of piglets directly after weaning. Akt Arb z artgem Tierhltg 2005, KTBL 441, 212-21

Ekesbo I (1981) Methods for the assessment of the environment from the point of view of animal welfare. Paper 32nd annual meeting of the EAAP, Zagreb, 318-91

Ekesbo I (1984) Methoden der Beurteilung von Umwelteinflüssen auf Nutztiere unter besonderer Berücksichtigung der Tiergesundheit und des Tierschutzes. Wien tierärztl Mschr 71, 186-90

Ekkel ED, van Doorn CEA, Hessing MJC, Tielen MJM (1995) The specific-stress-free housing system has positive effects on productivity, health, and welfare of pigs. J Anim Sci 73, 1544-51

Fraser D, Phillips PA, Thompson BK, Tennessen T (1991) Effect of straw on the behaviour of growing pigs Appl Anim Beh Sci 30, 307-18

Frerking H, Matschullat G, Müller E, Ikes D (1996) Tödliche Magengeschwüre - ständige Zunahme bei Schwein und Kalb. Tierärztl Umschau 51, 465-70

Gauly M, von Borell E, Hartung J, Hermann H-J, Hesse D, Hoppe S, Krieter J, Lüpping W, Mayer C, Moors E, Schulze V, Weiher O, Richter T (2006) Sachstandbericht der DGfZ e.V. - Projektgruppe »Prüfverfahren von Stalleinrichtungen und Aufstallungssystemen«. Züchtungskunde 78, 249-56

Gloor P (1984) Verletzungen und Veränderungen am Integument des Schweines als Indikatoren für die Tiergerechtheit eines Stallsystems. Akt Arb z artgem Tierhltg 1983, KTBL 299, 94-105

Gloor P (1988) Die Beurteilung der Brustgurtanbindung für leere und tragende Sauen auf ihre Tiergerechtheit unter Verwendung der »Methode Ekesbo« sowie ethologischer Parameter Schriftenr FAT 32, Tänikon

Grauvogl A (2000) Das Leben ist ein Spiel. Arch Tierz 43, 315-26

Greif G (1982) Grundlegende Untersuchungen zur Bemessung von Betonspaltenböden für Mastschweine unter besonderer Berücksichtigung der haltungsbedingten Gliedmaßenveränderungen. Diss Uni Gießen

Gygax L (2004) Statistische Modelle für ethologische Daten und Versuchsdesigns. Akt Arb z artgem Tierhltg 2003, KTBL 431, 53-61

Hagelsø Giersing M, Studnitz M (1996) Characterization and investigation of aggressive behaviour in the pig. Acta Agric Scand Sect A (Anim Sc) Suppl 27 (1996), 56-60

Hahn GL, Nienaber JA, DeShazer JA (1987) Air temperature influences on swine performance and behavior. Appl Engin Agric 3, 295-302

Hansen SKE, von Borell E (2000) Verhalten und Herzschlagvariabilität als Indikator für kurz- und langfristige Änderungen der Befindlichkeit von Schweinen durch regelmäßige GroomingSimulationen. Akt Arb z artgem Tierhltg 1999, KTBL 391, 81-9

Hesse D, Kukoschke B, Schlichting MC (1993) Verhalten von Mastschweinen in drei unterschiedlichen Einstreuhaltungsverfahren. Akt Arb z artgem Tierhltg 1992, KTBL 356, 177-89

Hörning B (1991) Artgemäße Tierhaltung - ein positiver Ansatz. Ökol Landbau 80, 40-2

Hörning B (1998) Tiergerechtheit und Tiergesundheit in ökologisch wirtschaftenden Betrieben. Dt tierärztl Wschr 105, 313-21

Hoy S, Müller K, Willig R (1997) Güllevermeidung durch das Kompoststallverfahren - eine Alternative? Umweltverträgliche Gülleaufbereitung und -verwertung, KTBL 242, 154-62

Jackisch T, Hesse D, Schlichting MC (1996) Raumstrukturbezug des Verhaltens von Mastschweinen in Haltungsverfahren mit und ohne Stroh. Akt Arb z artgem Tierhltg 1995, KTBL 373, 137-47

Jakob P (1987) Schweinemast im nichtwärmegedämmten Offenfrontstall auf Tiefstreue. SR FAT 28, Tänikon 
Janssens CJJG, Helmond FA, Wiegant VM (1995) The effect of chronic stress on plasma cortisol concentrations in cyclic female pigs depends on the time of day. Domestic Anim Endocrin 12, 167-77

Jensen KH, Pedersen BK, Pedersen LJ, Jørgensen E (1995a) Well-being in pregnant sows confinement versus group housing with electronic sow feeding. Acta Agric Scand Sect A (Anim Sci) 45, 266-75

Jensen KH, Pedersen LJ, Giersing Hagelsø AM, Heller KE, Jørgensen E, Ladewig J (1995b) Intermittent stress in pigs behavioural and pituitary-adrenocortical reactivity. Acta Agric Scand Sect A (Anim Sci) $45,276-85$

Jensen MB, Kyhn R (2000) Play behaviour in group-housed dairy calves, the effect of space allowance. Appl Anim Beh Sci 67, 35-46

Jensen P (1986) Observation on the maternal behaviour of free-ranging domestic pigs. Appl Anim Beh Sci $16,131-42$

Jensen P, Stangel G (1992) Behaviour of piglets during weaning in a semi-natural enclosure. Appl Anim Beh Sci 33, 227-38

Jensen P, Toates FM (1997) Stress as a state of motivational systems. Appl Anim Beh Sci 53, 145-56

Jones R, Nicol CJ (1998) A note on the effect of control of the thermal environment on the well-being of growing pigs. Appl Anim Beh Sci 60, 1-9

Kelly HRC, Bruce JM, Edwards SA, English PR, Fowler VR (2000) Limb injuries, immune response and growth performance of early-weaned pigs in different housing systems. Anim Sci 70, 73-83

Kendrick KM (2007) Quality of life and the evolution of the brain. Anim Welf 16 Suppl, 9-15

Krause M (1995) Verhalten und Körperschäden von Jungsauen in Gruppenhaltung bei simultaner oder sequentieller Futterzuteilung mit oder ohne Strohangebot. Diss TU München

Krötzl H, Sciarra C, Troxler J (1994) Der Einfluss von Rauhfutterautomaten, Strohraufen und Nagebalken auf das Verhalten von Mastschweinen. Akt Arb z artgem Tierhltg 1993, KTBL 361, 181-91

KTBL (Kuratorium für Technik und Bauwesen in der Landwirtschaft e.V., Hrsg.) (2006) Nationaler Bewertungsrahmen Tierhaltungsverfahren Methode zur Bewertung von Tierhaltungsanlagen hinsichtlich Umweltwirkungen und Tiergerechtheit. KTBL 446

Künzl C, Sachser N (2000) Auswirkungen der Domestikation auf Verhalten und endokrine Anpassungsreaktionen beim Meerschweinchen. Arch Tierz 43 SH, 153-8

Lay Jr DC, Haussmann MF, Daniels MJ, Harmon JD, Richard TL (2000) Swine housing impacts on environment and behavior a comparison between hoop structures and total environmental control Proc 1st ASAE (American Society of Agricultural Engineers) Int Conf Swine housing, Des Moines, IA, 9-11.10.2000, 49-53

Legge E (1992) Untersuchungen zum Verhalten frühabgesetzter Ferkel bei unterschiedlicher Gestaltung der Struktur des Buchtenbodens und zur Ermittlung repräsentativer Beobachtungszeiten. Diss Uni Bonn

Lindquist J-O (1974) Animal health and environment in the production of fattening pigs. Acta Vet Scand 15 Suppl 51, 1-78

Link M (1993) Untersuchungen zur Auswirkung der Stall- und Hüttenhaltung von tragenden und säugenden Sauen auf ausgewählte klinische und physiologische Merkmale. Diss TiHo Hannover

Losinger W, Bush EJ, Smith MA, Corso BA (1998) Mortality attributed to respiratory problems among finisher pigs in the United States. Prev Vet Med 37, 21-31

Lyons CAP, Bruce JM, Fowler VR, English PR (1995) A comparison of productivity and welfare of growing pigs in four intensive systems. Livest Prod Sci 43, 265-74

Maier P, Heizmann V, Reisenbauer K (1992) Sozialverhalten und Verhaltensontogenese von Hausschweinen in einem möblierten Familienstall. Akt Arb z artgem Tierhltg 1991, KTBL 351, 129-40

Manteuffel G, Puppe B (1997) Ist die Beurteilung der subjektiven Befindlichkeiten von Tieren möglich? Eine kritische Analyse aus naturwissenschaftlicher Sicht. Arch Tierz 40, 109-21

Marchant JN, Mendl MT, Rudd AR, Broom DM (1995) The effect of agonistic interactions on the heart rate of group-housed sows. Appl Anim Beh Sci 46, 49-56

Marchant JN, Rudd AR, Broom DM (1997) The effects of housing on heart rate of gestating sows during specific behaviours. Appl Anim Beh Sci 55, 67-78

Mason GJ (1991) Stereotypies a critical review. Anim Beh 41, 1015-37

Mason G, Mendl M (1993) Why is there no simple way of measuring animal welfare? Anim Welf 2, 301-19

Mayer C (1999) Stallklimatische, ethologische und klinische Untersuchungen zur Tiergerechtheit unter schiedlicher Haltungssysteme in der Schweinemast. Diss TU München, 1999

Mendl M (1999) Performing under pressure stress and cognitive function. Appl Anim Beh Sci 65, 221-44 
Mendl M, Paul ES (2005) Erfassen von Emotionen bei Tieren mittels eines kognitiven Ansatzes von Menschen zu Tieren und wieder zurück. Akt Arb z artgem Tierhltg 2005, KTBL 441, 9-20

Meyer H (2004) Schmerz, Angst und Leiden - Die belastenden Befindlichkeiten der Tiere. Akt Arb z artgem Tierhltg 2003, KTBL 431, 78-92

Meynhardt H (1990) Schwarzwild-Report mein Leben unter Wildschweinen Neumann, Leipzig

Morton DB, Griffiths PHM (1985) Guidelines on the recognition of pain, distress and discomfort in experimental animals and an hypothesis for assessment. Vet Rec 116, 431-6

Nielsen EK, Ingvartsen L (2000) Effects of cereal disintegration method, feeding method and straw bedding on stomach characteristics including ulcers and performance in growing pigs. Acta Agric Scand, A Anim Sci 50, 30-38

Otten W, Puppe B, Stabenow B, Kanitz E, Schön PC, Brüssow KP, Nürnberg G (1997) Agonistic interactions and physiological reactions of top- and bottom-ranking pigs confronted with a familiar and an unfamiliar group preliminary results. Appl Anim Beh Sci 55, 79-90

Otten W, Kanitz E, Tuchscherer M (2000) Prenatal stress in pigs effects on growth, physiological stress reactions and immune function. Arch Tierz $43 \mathrm{SH}, 159-64$

Peeters E, Driessen B, Moons CPH, Ödberg FO, Geers R (2006) Effect of temporary straw bedding on pigs' behaviour, performance, cortisol and meat quality. Appl Anim Beh Sci 98, 234-48

Pflanz W (2007) Gesamtheitliche Beurteilung innovativer Schweinemastverfahren für BadenWürttemberg. Diss Uni Hohenheim

Puppe B, Ernst K, Schön PC, Manteuffel G (2005) Aufmerksamkeit, Aktivität und positive kognitive Bewertung beim Futtererwerb von Schweinen - experimenteller Ansatz und Effekte auf Verhalten und Gesundheit. Akt Arb z artgem Tierhltg 2005, KTBL 441, 21-30

Puppe B, Ernst K, Schön PC, Manteuffel G (2007) Cognitive enrichment affects behavioural reactivity in domestic pigs. Appl Anim Beh Sci 105, 75-86

QS (Qualität und Sicherheit GMBH) (2009) Leitfaden Schlachtung/Zerlegung, Version 01.01.2009, Status Freigabe, http://www.q-s.info/fileadmin/QS_Fileadmin/downloads/FleischFleischwarenUndFuttermittel/ Schlachtung_Zerlegung/Leitfaden_Checkliste_Schlachtung_Zerlegung/lf_sz_d_frei_090101.pdf, 63-72 [Zugriff am 07.07.2009]

Rinaldo D, Le Dividich J (1991) Assessment of optimal temperature for performance and chemical body composition of growing pigs. Livest Prod Sci 29, 61-75

Rist M (1978) Gesundheit als gelungenes Wechselspiel zwischen In- und Umwelt Ber 1 AGHST (Arbeitsgemeinschaft Gesunde Haltungstechnik und Stallbau)-Arbeitstagung, BVA (Bundesversuchsanstalt für alpenländische Landwirtschaft) Gumpenstein 3.-4.10.1978, 25-29

Robert S, Rushen J, Farmer C (1997) Both energy content and bulk of food affect stereotypic behaviour, heart rate and feeding motivation of female pigs. Appl Anim Beh Sci 54, 161-71

Rushen J (1991) Problems associated with the interpretation of physiological data in the assessment of animal welfare. Appl Anim Beh Sci 28, 381-6

Rushen J (1993) The »coping « hypothesis of stereotypic behaviour. Anim Beh 45, 613-5

Rushen J, de Pasillé AMB, Schouten W (1990) Stereotypic behavior, endogenous opioids, and postfeeding hypoalgesia in pigs. Physiol Behav 48, 91-6

Rushen J, Schwarze N, Ladewig J, Foxcroft G (1993) Opioid modulation of the effects of repeated stress on ACTH, cortisol, prolactin, and growth hormone in pigs. Physiol Behav 53, 923-8

Sambraus, HH (985) Indikatoren und Auswirkungen nicht tiergerechter Haltungssysteme. Vortrag (paper), Tierzuchtsem und Tierzuchttagung BAL (Bundesanstalt für alpenländische Landwirtschaft) Gumpenstein, Irdning 13.-15.05.1985 und 11.-12.06.1985

Savary P, Gygax L, Hauser R, Wechsler B, Jungbluth T (2005) Auswirkungen einer Kunststoffplatte im Liegebereich auf das Liegeverhalten und Veränderungen am Integument bei Mastschweinen. Akt Arb z artgem Tierhltg 2005, KTBL 441, 59-67

Schäfer-Müller K (1996) Untersuchungen zur Gruppenhaltung tragender Sauen unter besonderer Berücksichtigung des Einflusses von Stroh auf Leistung, Konstitution und Verhalten. Diss Uni Kiel, SR Inst Tierzucht und Tierhaltung, Univ Kiel 87

Schimmel D (1992) Respiratorische Erkrankungen des Schweines und ihre Bedeutung beim Aufbau gesunder Tierbestände. Bundesgesundheitsblatt 35, 392-3

Scholz K (1981) Serumlipide, Serumproteine und Metabolite des Proteinstoffwechsels beim Göttinger Minischwein. Normalwerte und genetische Parameter. Diss Uni Göttingen

Schönreiter S, Zanella AJ (2000) Assessment of cortisol in swine by saliva new methodological approaches. Arch Tierz 43 SH, 165-70 
Schuster H (1984) Verhalten und Klauengesundheit von frühabgesetzten Ferkeln in Flatdecks mit verschiedenen Bodenausführungen. Diss Uni Hohenheim

Seufert H, Jungbluth T, Greif G (1980) Zur Eignung perforierter Böden für die Schweinehaltung. Landtechnik 35, 404-8

Siard N, Štuhec I, Ladewig J, Schlichting MC (1997) Correlation between plasma and salivary cortisol in growing pigs. Akt Arb z artgem Tierhltg 1996, KTBL 376, 199-206

Siegel HS (1987) Effects of behavioural and physical stressors on immune response. In: Wiepkema PR, van Adrichem PWM (ed) (1987) Biology of stress in farm animals: an integrative approach. Martinus Nijhoff, Dordrecht, 39-54

Siegwart R, Wechsler B, Gygax L (2005) Wird das Wohlbefinden von Mastbullen auf gummierten Spaltenböden mit vergrössertem Platzangebot erhöht? Akt Arb z artgem Tierhltg 2005, KTBL 441, 76-85

Signoret JP, Vieuille C (1996) Effectiveness and limitations of physiological versus ethological criteria to assess the welfare of pigs in relation to the housing system. Pig News and Info 17, 115N-21N

Simonsen HB (1990) Behaviour and distribution of fattening pigs in the multi-activity pen. Appl Anim Beh Sci 27, 311-24

Sommer B, Leeb C, Troxler J, Schuh M (1999) Erstellung eines praxistauglichen Schlüssels zur Beurteilung von Haltungssystemen tragender Zuchtsauen auf Tiergerechtheit anhand von Integumentveränderungen. In: Proc 14. IGN 6. Freiland Tagung Tierhaltung und Tiergesundheit, Wien 29.9.-1.10.1999, 116-9

Spoolder HAM, Burbidge JA, Edwards SA, Simmins PH, Lawrence AB (1995) Provision of straw as a foraging substrate reduces the development of excessive chain and bar manipulation in food restricted sows. Appl Anim Beh Sci 43, 249-62

Stolba A (1984) Verhaltensmuster von Hausschweinen in einem Freigehege Bemerkungen zum Film. Akt Arb z artgem Tierhltg 1983, KTBL 299, 106-16

Stolba A, Wood-Gush DGM (1984) The identification of behavioural key features and their incorporation into housing design for pigs. Ann Rech Vét 15, 287-98

Stolba A, Wood-Gush DGM (1989) The behaviour of pigs in a semi-natural environment. Anim Prod 48, 419-25

Stubbe A (2000) Entwicklung und Beurteilung einer Beschäftigungstechnik für Mastschweine in intensiven Haltungssystemen. Diss Uni Hohenheim

Terlouw EMC, Lawrence AB, Ladewig J, de Passille AM, Rushen J, Schouten WGP (1991) Relationship between plasma cortisol and stereotypic activities in pigs. Behav Processes 25, 133-53

Terlouw EMC, Schouten WGP, Ladewig J (1997) Physiology. In: Appleby MC, Hughes BO (ed) Animal Welfare, CAB Int, Wallingford, 143-58

Teuchert-Noodt G (2004) Neurotransmitter und Befindlichkeiten im Tier/Mensch-Vergleich. Akt Arb z artgem Tierhltg 2003, KTBL 431, 9-19

Tuchscherer M, Manteuffel G (2000) Die Wirkung von psychischem Stress auf das Immunsystem. Ein weiterer Grund für tiergerechte Haltung (Übersichtsreferat). Arch Tierz 43, 547-60

Turner SP, Ewen M, Rooke JA, Edwards SA (2000) The effect of space allowance on performance, aggression and immune competence of growing pigs housed on straw deep-litter at different group sizes. Livest Prod Sci 66, 47-55

Van de Weerd HA, Docking CM, Day JEL, Breuer K, Edwards SA (2006) Effects of species-relevant environmental enrichment on the behaviour and productivity of finishing pigs. Appl Anim Beh Sci 99, 230-47

Van Dijk AJ, Van Engen MJ, Hemke G (2000) Assessment of the daily distribution of oral stereotypic behaviour of group housed pregnant sows sequentially fed by an electronic sow feeder station. Vortrag (paper) 51st Ann Meet EAAP (European Association for Animal Production), Den Haag 21.24.08.2000

Van Rooijen J (1984) Impoverished environments and welfare. Appl Anim Beh Sci 12, 3-13

Van Rooijen J (1991) Predictability and boredom. Appl Anim Beh Sci 31, 283-7

Velarde A, Geers R (ed) On farm monitoring of pig welfare. Wageningen Academic Publishers, Wageningen

Verstegen MWA, Brascamp EW, van der Hel W (1978) Growing and fattening of pigs in relation to temperature of housing and feeding level. Can J Anim Sci 58, 1-13

Vieuille-Thomas C, Le Pape G, Signoret JP (1995) Stereotypies in pregnant sows indicators of influence of the housing system on the patterns expressed by the animals. Appl Anim Beh Sci 44, 19-27

Von Borell E (2000) Stress and coping in farm animals. Arch Tierz 43 SH, 144-52 
Von Borell E, Johnson RW, Anderson LL (1995) Neurobiologische Untersuchungen zum Verhalten von Schweinen unter Belastung. Akt Arb z artgem Tierhltg 1994, KTBL 370, 171-7

Weber REF (2003) Wohlbefinden von Mastschweinen in verschiedenen Haltungssystemen unter besonderer Berücksichtigung ethologischer Merkmale. Diss Uni Hohenheim

Weber REF, Valle Zárate A (2005) Der Begriff Wohlbefinden in der Nutztierhaltung - Diskussion aktueller Definitionsansätze als Grundlage für praxisorientierte Forschung am Beispiel Mastschweinehaltung . Arch Tierz 48, 475-89

Wechsler B (1992) Ethologische Grundlagen zur Entwicklung alternativer Haltungsformen. Schweizer Arch Tierheilk 134, 127-32

Wechsler B (2003) Optimierung der Versuchsplanung bei ethologischen Untersuchungen. Akt Arb z artgem Tierhltg 2003, KTBL 431, 43-52

Wechsler B (2005) An authorisation procedure for mass-produced farm animal housing systems with regard to animal welfare. Livest Prod Sci 94, 71-9

Wemelsfelder $F$ (1997) Life in captivity its lack of opportunities for variable behaviour. Appl Anim Beh Sci 54, 67-70

Wiepkema PR, Schouten WGP, Koene P (1993) Biological aspects of animal welfare new perspectives. J Agric Env Ethics, 6 Suppl 1-2, 93-103

Witte $F$ (1999) Untersuchungen zur Tiergesundheit von Mastschweinen - unter besonderer Berücksichtigung der Klauengesundheit - in drei Betrieben mit unterschiedlichen Aufstallungsarten in Hessen. Diss Uni Gießen

Zanella AJ, Broom DM (1993) Endogene Opioide und Indikatoren für tierschutzrelevante Anpassungs vorgänge. Akt Arb z artgem Tierhltg 1992, KTBL 356, 116-26

Zanella AJ, Broom DM, Mendl M (1991) Responses to housing conditions and immunological state in sows. Anim Prod 52, 579

ZDS (Zentralverband der deutschen Schweineproduktion, Hrsg) (1998) Zahlen aus der deutschen Schweine produktion

ZDS (Zentralverband der deutschen Schweineproduktion, Hrsg) (2005) Schweineproduktion 2004 in Deutschland

Corresponding author:

Dr. RAGNHILD E.F. WEBER-JONKHEER

email: reggae_w@yahoo.com

Langwartweg 99, 53129 Bonn, Germany 